PALEO

Revue d'archéologie préhistorique

$28 \mid 2017$

Varia

\title{
Marcel Jeannet (1935-2017)
}

Marie-Francoise Bonifay, Alain Argant, Jacqueline Argant et Jean-Philip Brugal

\section{OpenEdition}

Journals

Édition électronique

URL : http://journals.openedition.org/paleo/3070

DOI : $10.4000 /$ paleo.3070

ISSN : 2101-0420

Éditeur

SAMRA

Édition imprimée

Date de publication : 30 décembre 2017

Pagination : 17-24

ISSN : 1145-3370

Référence électronique

Marie-Francoise Bonifay, Alain Argant, Jacqueline Argant et Jean-Philip Brugal, « Marcel Jeannet

(1935-2017) », PALEO [En ligne], 28 | 2017, mis en ligne le 01 juin 2018, consulté le 14 septembre 2020. URL : http://journals.openedition.org/paleo/3070

\section{(c) (i) (2) $\Theta$}

PALEO est mis à disposition selon les termes de la licence Creative Commons Attribution - Pas d'Utilisation Commerciale - Pas de Modification 4.0 International. 


\section{Marcel JEANNET $(1935-2017)$}

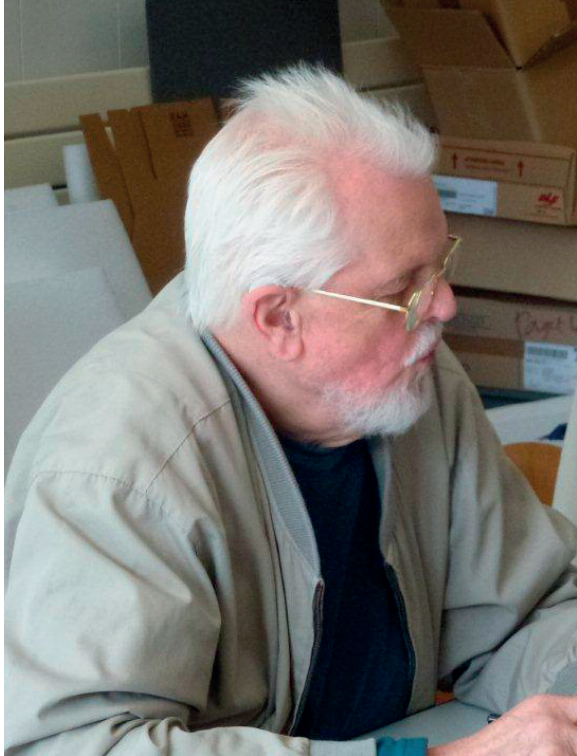

(Photo J.-Ph. Brugal, avril 2014)

\section{Quelques Témoignages et éléments biographiques}

Marcel Jeannet faisait partie de ces chercheurs qui ont défriché la Paléontologie du Quaternaire en France ; spécialiste des micromammifères, il a permis aux études ultérieures d'émerger.

Pour donner une idée de l'importance accordée dans les années 1960-1970 à l'étude des faunes quaternaires, je ferai référence à mon histoire personnelle : le Professeur Jean Piveteau, mon Directeur de thèse, m'avait demandé de travailler sur l'ensemble des grandes faunes quaternaires du sud de la France...Il fut très étonné lorsque deux années plus tard je vins lui demander de restreindre le sujet aux seuls carnivores : débordée par les fossiles et la bibliographie en langue étrangère dus à la découverte de sites antérieurs au Paléolithique supérieur, je venais de mettre les pieds dans un monde quasiment ignoré en France et peu connu à l'extérieur.

Il en a été de même pour Marcel Jeannet.

Mais l'étude du Pléistocène européen n'est pas facile. II est difficile de comparer les faunes de sites éloignés géographiquement car les changements climatiques, maintenant bien mieux connus, ne se font pas de façon homogène sur les courtes périodes que nous étudions. Les comparaisons anatomiques, bases acceptées de notre travail de paléontologue, aboutissaient à des comparaisons aléatoires et, rapidement, la compréhension des paléoenvironnements (avec toutes les difficultés que cela comporte) nous est devenue indispensable.

Il en a été de même pour Marcel Jeannet.

Enfin, si mes collègues et moi-même avions tout notre temps pour réfléchir, lire, traduire, lui, non. Sans jamais se plaindre, il occupait un emploi à temps complet, nous permettant de comprendre ce qu'était la passion de la Paléontologie... ajoutée à une gentillesse et un sens de l'humour que j'ai toujours appréciés.

Merci Marcel Jeannet.

Marie-Francoise BONIFAY 
Passionné depuis toujours par les cailloux (Yvette dixit), il fait partie très tôt de l'équipe "Jean Combier-André Jeannet" et c'est à Orgnac que nous le rencontrons pour la première fois en 1966. Les fouilles commencent sur le site d'Orgnac 3 nouvellement protégé et équipé. Marcel devient alors responsable du tamisage et du tri de la microfaune. Nous le revoyons assis en tailleur fouillant les tamis, imperturbable sous le soleil ! Et ce sont les Rongeurs de ce gisement qui feront l'objet de son DES de Sciences naturelles huit ans plus tard, au prix d'un travail gigantesque. Avec une puissance de travail souvent à la limite de la démesure, il travaille de nuit au tri postal et étudie pendant l'après-midi, perdant la notion du temps et se reposant totalement sur son épouse Yvette pour assurer les tâches familiales essentielles. Le jeu des jours de compensation lui permet de dégager de longues périodes de congé qu'il consacre entièrement à sa recherche. Il prend sa retraite en 1990 et dès 1991 il adhère à l'ARPA (Association de Recherche Paléoécologique en Archéologie) qui vient d'être créée et dont il sera secrétaire jusqu'en 2015, poste qu'il abandonne alors pour raisons de santé. Cette association lui a permis de continuer son travail dans des conditions techniques et matérielles satisfaisantes. II a largement contribué au développement et au rayonnement de l'ARPA en réalisant les analyses d'au moins 74 gisements en lien avec une cinquantaine de chercheurs. Nous garderons aussi le souvenir de son humour caustique en toutes circonstances et en particulier lors des assemblées générales.

II faut dire aussi que c'était un grand sportif : basket au Club de Charnay, puis cyclotourisme à un haut niveau (club Charnay Cyclo) qui l'amène à gravir souvent en solitaire tous les cols des Alpes dont le Grossglöchner ! Marcel ne faisait rien à moitié, le cyclo a pendant un temps éclipsé les Rongeurs !

Alain et Jacqueline ARGANT

Une ombre passe, et de manière subtile un grand monsieur s'en est allé. Marcel Jeannet nous a quitté le 4 octobre 2017 et laisse derrière lui plein de beaux souvenirs aux uns et aux autres, ainsi qu'un ensemble de travaux conséquents et diversifiés en Paléontologie et Paléoécologie du Quaternaire. Autodidacte, et par profession contrôleur divisionnaire à La Poste, il se passionne très tôt pour la Préhistoire et commence à fouiller avec son frère André (site de Vergisson-les-Tanières à la fin des années soixante). II se spécialise peu à peu en Zoologie et Paléontologie, et ce sont les microfaunes qui l'attirent, amenant toute sa famille ramasser des pelotes de rapaces remplies de restes osseux qu'il dépiaute avec plaisir dans sa région du Mâconnais et de Rhône-Alpes. II en avait - des pelotes - de toute la France. Ses contacts avec les équipes de Paléontologie de Lyon et de Dijon lui permettent de soutenir le 19 juin 1974 un Diplôme d'Études supérieures de Sciences naturelles, Université de Dijon, Institut des Sciences de la Terre (Centre de Paléogéographie et de Paléobiologie Évolutives), sous la direction de Jean Chaline, sur les rongeurs d'Orgnac 3 en Ardèche (il n'avait pas le Bac !). II était rattaché depuis plusieurs années à notre équipe, à l'UMR 7269 LAMPEA d'Aix-en-Provence.

Marcel adorait les petits vertébrés, les petites « choses », et en particulier les rongeurs. Ses collections, modernes et fossiles, sont impressionnantes, autant que sa bibliothèque, très diversifiée, curieux de tout... son appartement débordait (plus le garage et la cave !), au grand dam d'Yvette, son épouse, qui l'a cependant toujours soutenu et accompagné parfois sur le terrain. II râlait « j'ai plus de place ! »...

Je le vois encore (il était de toutes les campagnes de Coudoulous), toujours discret, courbé sur ses tamis, à faire sécher les sédiments, puis penché sur sa bino pour isoler et prélever soigneusement, par touches délicates, les plus petits restes osseux et dentaires pour les identifier, les mesurer, les compter... et les ranger dans ces petits piluliers bien inventoriés, avec de très petites étiquettes, de son écriture serrée... de tous les groupes microfauniques (rongeurs, insectivores, chiroptères, petits carnivores, oiseaux, amphibiens, reptiles, oiseaux... jusqu'à la coquille interne de limace !), avec un enthousiasme et une patience remarquable.

C'était un grand spécialiste des Rongeurs (voir sa bibliographie ci-dessous) et un grand anatomiste (il était parfois capable d'identifier du postcrânien) ; il a décrit de nouvelles espèces, précisé leur évolution et leur apport pour la biochronologie, mis au point une méthode d'écologie quantifiée performante (2010) permettant d'inférer de nombreux paramètres climatiques, et de corréler ces résultats avec les courbes isotopiques. Par sa compétence, largement reconnue, il était très sollicité et a de fait participé à de très nombreux programmes de recherches, de l'Université, du CNRS ou du Ministère de la Culture ; il intervenait sur de nombreux chantiers de fouilles, prenant en charge la recherche de microfaune et initiant de nombreux étudiants dont certains deviendront des disciples. II aimait partager et se retrouver parmi ces équipes de fouilles discutant Science, et du reste... Sa production scientifique démontre la diversité et la richesse de son travail, et son implication sur des sites prestigieux (l'Escale, Lunel-Viel, Orgnac, Coudoulous, Baume Néron, Mandrin, Les Fieux, Solutré...) en France, mais aussi d'Italie ou du Portugal. Son dernier article paru en début d'année (Paleo, 2016) traitait des muridés de l'Igue des Rameaux (Tarn et Garonne), site qui m'est cher, et me fait penser à François Rouzaud... de belles campagnes de fouilles sur le Causse, avec François et Marcel, et toute l'équipe. 
Amateur de bon vin, humour fin et pétillant, œil malicieux sous ses lunettes (j'allais dire bésicles) pour sortir un bon mot ou débattre de rat taupier... Marcel alliait une grande érudition, restant toujours extrêmement précis et soigneux, avec une grande humilité et beaucoup d'esprit. C'était une perle dans son genre, et nul doute que notre communauté accusera ce manque... pour un certain temps. J'avais, personnellement, beaucoup d'amitié et d'affection pour lui.

Nous nous rappellerons sa signature que je reproduis ci-dessous... petite chose mais révélant une grande âme. Bon vent Monsieur Marcel, Cher Collègue, Cher Ami, au pays des gros rongeurs...

Jean-Philip BRUGAL

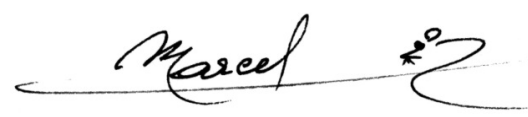

\section{Publications de Marcel Jeannet (1964-2017)}

JEANNET M. 1964 - Découverte de céramique de l'Age du fer à Préty (Saône-et-Loire), Revue archéologique de l'Est et du Centre-Est, p. 153-155.

JEANNET M. 1964 - Une fibule hallstattienne découverte à Préty, Saône-et-Loire, Revue archéologique de l'Est et du Centre-Est, p. 131-140.

JEANNET M. 1970 - Le gisement moustérien de Vergisson : Les Tasnières (Saône-et-Loire), Revue archéologique de l'Est, t. 3-4, p. 343-367.

JEANNET M. 1971 - Découverte d'une dent de mammouth à Vaulx-en-Velin (Rhône), Bulletin mensuel de la Société linnéenne de Lyon, t. 40, 5, p. 65-67.

JEANNET M. 1971 - Préhistoire et archéologie : Le tertre de Belleville. In: Bulletin mensuel de la Société linnéenne de Lyon, 40 année, $n^{\circ}$ 3, mars 1971. p. 42-44.

JEANNET M. 1974 - Les Rongeurs d'Orgnac 3 (Ardèche). Diplôme d'Études supérieures de Sciences naturelles, Université de Dijon, Institut des Sciences de la terre (Centre de Paléogéographie et de Paléobiologie Évolutives), sous la direction de Jean Chaline. Jury : P. Rat, H. Tintant, J. Chaline, J. Combier (invité).

JEANNET M. 1974 - Pliomys chalinei nov. sp. (Arvicolidae, Rodentia) du Pléistocène moyen d'Orgnac 3 (Ardèche), Bulletin de la Société géologique de France, p. 1-2.

JEANNET M. 1974 - Contribution à l'étude des formations superficielles des environs de Lacrost (Saône-et-Loire), Bulletin trimestriel de la Société d'histoire naturelle de Mâcon, t. 25, p. 31-45.

JEANNET M. 1976 - Lunel-Viel, Nouvelles Archives du Museum d'Histoire naturelle de Lyon, t. 14, suppl., p. 45-46.

JEANNET M. 1977 - Recherche de microfaune à la Grotte de l'Escale, Saint-Estève-Janson (Bouches-du-Rhône) : campagne 1977, Nouvelles Archives du Museum d'Histoire naturelle de Lyon, t. 15, suppl., p. 53-.
JEANNET M. - 1977, Recherche de microfaune à la Rochelambert, Saint-Paulien (Haute-Loire) : campagne 1977, Nouvelles Archives du Museum d'Histoire naturelle de Lyon, t. 15, suppl., p. 49-52.

JEANNET M. 1978 - L'hypsodontie chez les Campagnols et les autres mammifères. In: Bulletin mensuel de la Société linnéenne de Lyon, 47 année, $n^{\circ}$ 5, mai 1978. p. 206-213

JEANNET M. 1978 - Recherches sur les rongeurs quaternaires en 1978, Nouvelles Archives du Museum d'Histoire naturelle de Lyon, t. 16, suppl., p. 71-74.

CANTET M. ( ), CLOT A., GEAY A., GRANIER J., JEANNET M., MARSAN G., MOURER C., RAGE J-C. 1978 - Le gisement du Paléolithique supérieur de la Brette. 2 à Condom (Gers). Bulletin de la Société d'Histoire naturelle de Toulouse, 114, 3-4 : 332-359.

JEANNET M. 1979 - Les rongeurs et l'environnement au Mont des Espélugues (Lourdes, Hautes-Pyrénées), Bulletin de la Société d'Histoire naturelle de Toulouse, t. 115, 1-2, p. 53-64.

JEANNET M. 1979 - Note sur quelques éléments de microfaune reçus en 1979, Nouvelles Archives du Museum d'Histoire naturelle de Lyon, t. 17, suppl., p. 59-64.

GIACOBINI G., MALARODA R., BIANCOTTI A., JEANNET M., Arobba D. 1980 - La breccia ossifera del Monte dei Cros (Andonno, Cuneo), In: Studi di archeologia dedicati a Pietro Barocelli, Torino, Soprintendenza archeologica del Piemonte, p. 55-73.

JEANNET M. 1980 - Les rongeurs de quelques sites holocènes (Vallon-Pont-d'Arc et Foissac), würmiens (Gréolières, Casteljau et Bendorf) et rissien (Azé), Nouvelles Archives du Museum d'Histoire naturelle de Lyon, t. 18, suppl., p. 29-34.

JEANNET M. 1981 - Les rongeurs du gisement acheuléen d'Orgnac 3 (Ardèche). Essai de paléoécologie et de chronostratigraphie. In : Bulletin mensuel de la Société linnéenne de Lyon, 50 année, $n^{\circ} 2$, février 1981. pp. 49-71

HERRENSCHMIDT V., AUPETIT P., JEANNET M., LIBOIS R.M., PHILIPPE M.-A., ROSOUX R., SAINT- 
GIRONS M.-C., TURPIN F. 1983 - Les mammifères du Marais poitevin : le régime des carnivores, Annales de la Société des Sciences naturelles de la Charente-maritime, t. 7, 1, p. 101-104.

ALLAIN J., DESBROSSE R., KOZLOWSKI J.K., RIGAUD A., avec la collaboration de, JEANNET M., LEROIGOURHAN A. 1985 - Le Magadalénien à navettes, Gallia Préhistoire, t. 28, 1, p. 37-124.

JEANNET M. 1985 - Sur la présence de Microtus cabrerae (Arvicolidae, Rodentia) dans les Pyrénées-Orientales (France). In: Bulletin mensuel de la Société linnéenne de Lyon, 54 année, $n^{\circ} 5$, mai 1985. pp. 120-121.

DEFLEUR A., BEZ J.-F., CRÉGUT-BONNOURE E., FONTUGNE M., JEANNET M., MAGNIN F., TALON B., THINON M., COMBIER J. 1994 - Industries, biostratigraphie, restes humains et datation du gisement moustérien de la Baume Néron (Soyons,Ardèche), Comptes rendus de l'Académie des sciences (2), t. 318, p. 1409-1414.

JEANNET M. 1995 - Les Cricétidés (Mammalia, Rodentia) des gisements moustériens de Soyons (Ardèche, France). In : Bulletin mensuel de la Société linnéenne de Lyon, 64 année, $n^{\circ} 1$, janvier 1995. pp. 41-48.

BRUGAL J.-P., COSTAMAGNO S., ELLWOOD B.B., JAUBERT J., JEANNET M., KERVAZO B., THERY I. 1996 - Tour de Faure. Coudoulous II, Bilan scientifique. Région Midi-Pyrénées, t. 1996, p. 137-139.

JEANNET M., ALLARD M., JUILLARD F. 1996 - Première découverte de Galemys pyrenaicus (Insectivora, Desmaninae) dans le Quaternaire français, Revue de Paléobiologie, t. 15, 1, p. 205-213.

BRUGAL J.-P., COSTAMAGNO S., ELLWOOD B.B., JAUBERT J., JEANNET M., KERVAZO B., THERY I. 1997 Tour de Faure. Coudoulous II, In: Bilan scientifique de la région Midi-Pyrénées 1996, Paris, Direction régionale des Affaires culturelles Midi-Pyrénées - Service régional de l'Archéologie / Ministère de la Culture et de la Francophonie - Direction du Patrimoine - Sous-Direction de l'Archéologie, p. 137-139.

JAUBERT J., BRUGAL J.-P., JEANNET M., KERVAZO B., MOURRE V. 1997 - Tour de Faure. Coudoulous I, Bilan scientifique. Région Midi-Pyrénées, t. 1996, p. 135-137.

BINTZ P., avec les contributions de, AFFOLTER J., ARGANT J., BERNARD-GUELLE S., BUI THI MAI, BRESSY C., COUSSERAN S., GIRARD M., JEANNET M., MALENFANT M., MONIN G., PELLETIER D., PICAVET R., PHILIBERT S. (Dir.) 1998 - Mésolithique et processus de néolithisation dans les Alpes du nord. Peuplement et paléoenvironnement. Programme collectif de recherche. Rapport 1998, Grenoble / Aix-en-Provence, ERPPA / ESEP, n. p. [consulté le 23.03.99].

GIRAUD Y., BRUGAL J.-P., JEANNET M. 1998 - Un nouveau gisement moustérien en moyenne vallée du Rhône : la grotte Mandrin à Malataverne (Drôme), Bulletin de la Société préhistorique française, t. 95, 1, p. 7-15.
BINTZ P., avec les contributions de ARGANT J., BEECHING A., BERNARD C., BRESSY C., BRISOTTO V., CHAIX L., COUSSERAN S., JEANNET M., MORIN A., NATON H.-G., PELLETIER D., THIÉBAULT S., THIRAULT E., TOMÉ C., et la collaboration de CORBINEAU J., MONIN G., WESTPHAL A. (Dir.) 1999 - Les sites archéologiques de Aulp-du-Seuil à Saint-Bernard du Touvet (Chartreuse, Isère). L'abri sous bloc $n^{\circ} 1$ : rapport scientifique 1998. L'abri sous bloc $n^{\circ} 2$ : raport de sondage 1998, Grenoble / Aix-en-Provence, ERPPA / ESEP, 113 p. [consulté le 23.03.99] .

DEBARD E., FAURE M., GUÉRIN C., ARGANT J., JEANNET M. 1999 - Le gisement pléistocène de l'aven Flahaut à Saint-Remèze (Ardèche), Ardèche Archéologie, t. 16 , p. 1-6.

JAUBERT J., BRUGAL J.-P., BISMUTH T., COLONGE D., JEANNET M., KERVAZO B., MOURRE V. 1999 - Tour de Faure. Coudoulous I, Bilan scientifique. Région MidiPyrénées, t. 1998, p. 150-152.

JEANNET M. 2000 - Gruta da Figueira Brava : les Rongeurs, Memórias da Academia das Ciências de Lisboa. Classe de ciências, t. 38, p. 179-243.

JEANNET M. 2000 - Biométrie et écologie de Microtus brecciensis (Mammalia, Rodentia), Revue de Paléobiologie, t. 19, 2, p. 339-357.

JEANNET M., CARTONNET M. 2000 - La microfaune de la Chênelaz (Hostias, Ain) : l'environnement et son influence sur la biométrie dentaire chez Arvicola terrestris (Rodentia, Mammalia), Revue de Paléobiologie, t. 19, 2, p. 475-492.

BINTZ P., avec les contributions de GRIGGO C., JEANNET M., MORIN A., PELLETIER D. 2001 - L'abri sous bloc $n^{\circ} 2$ de l'Aulp du Seuil à Saint-Bernard-du-Touvet (Chartreuse, Isère). Rapport de fouille 2000, in: Les sites archéologiques de l'Aulp du Seuil à Saint-Bernard-duTouvet (Chartreuse, Isère), Pelletier D., Bintz P. (Dir.), Grenoble, UMR 6636 - CNRS - Institut Dolomieu Université Joseph Fourier, p. 31-48.

BRUGAL J.-P., COCHARD D., ELLWOOD B.B., GIRARD M., JAUBERT J., JEANNET M., KERVAZO B., LOUCHART A., QUINIF Y., THÉRY I. 2001 - Tour-deFaure : Coudoulous II, Bilan scientifique. Région MidiPyrénées, t. 2000, p. 126-128.

JAUBERT J., BRUGAL J.-P., JEANNET M., KERVAZO B., MOURRE V. 2001 - Tour-de-Faure : Coudoulous I, Bilan scientifique. Région Midi-Pyrénées, t. 2000, p. 123-126.

JEANNET M. 2001 - La microfaune et l'environnement de la grotte du Noisetier à Fréchet (Hautes-Pyrénées), Préhistoire ariégeoise, t. 56, p. 83-90.

JEANNET M. 2001 - La petite faune in Le creux des Elaphes (commune des Déserts, plateau du Revard, Parc naturel régional du Massif des Bauges, Savoie) / M. Philippe (Coord.), Cahiers scientifiques du Muséum d'histoire naturelle de Lyon, t. 1, p. 39-45. 
PELLETIER D., BINTZ P., avec les contributions de GRIGGO C., JEANNET M., MORIN A., NATON H.-G., et la participation technique de BERNARD C. 2001 - Les sites archéologiques de l'Aulp du Seuil à Saint-Bernard-duTouvet (Chartreuse, Isère), Grenoble, UMR 6636 - CNRS Institut Dolomieu - Université Joseph Fourier, 69 p. [consulté le 2001.04.05]

PHILIPPE M., CHAIX L., GUIBOT S., HOBLÉA F., JEANNET M., VALLI A. 2001 - Le creux des Elaphes (commune des Déserts, plateau du Revard, Parc naturel régional du Massif des Bauges, Savoie), Cahiers scientifiques du Muséum d'histoire naturelle de Lyon, t. 1, p. 19-105.

JEANNET M. 2002 - Chapitre 12 : microfaune et environnement au Crot du Charnier à Solutré, in: Solutré 1968-1998, Combier J., Montet-White A. (Dir.), Paris, Société préhistorique française, p. 169-180 (Mémoires de la Société préhistorique française ; 30 ).

JEANNET M. 2002 - Sur des pelotes d'Effraie recueillies à Igé (Saône et Loire), Bulletin scientifique de Bourgogne, t. 50,2 , p. 30-36.

FONTANA L., LANG L., CHAUVIÈRE F.-X., JEANNET M., MAGOGA L. 2003 - Nouveau sondage sur le site paléolithique des Petits Guinards à Creuzier-le-Vieux (Allier, France) : des données inattendues, Bulletin de la Société préhistorique française, t. 100, 3, p. 591-596.

FONTANA L., LANG L., CHAUVIÈRE F.-X., JEANNET M., MOURER-CHAUVIRÉ C., MAGOGA L. 2003 Paléolithique supérieur récent du nord du Massif central : des données inattendues sur le site des Petits Guinars à Cruzier-le-Vieux (Allier, France), Préhistoire du Sud-Ouest, t. 10, p. $77-93$

JAUBERT J., KERVAZO B., BRUGAL J.-P., FALGUĖRES C., JEANNET M., LEMORINI C., LOUCHART A., MAKSUD F., MARTIN H., MOURRE V., QUINIF Y., THIÉBAUT C. 2003 - La séquence Pléistocène moyen de Coudoulous I (Lot) : bilan pluridisciplinaire, In: Colloque international - 22-25 septembre 200, Rennes, Université de Rennes 1: Données récentes sur les modalités de peuplement [...] [volume des résumés], UMR 6566 du CNRS, UMR 6569 du CNRS (Dir.), Rennes, UMR 6566 du CNRS « Civilisations atlantiques et archéosciences », p. 51-53.

JEANNET M., LABE B. 2004 - Découverte fortuite de microfaune à Saint-Pierre des Tripiers (Lozère), La Cave des Moineaux, t. 15, septembre, p. 56-58.

JAUBERT J., BRUGAL J.-P., avec la collaboration de JEANNET M., KERVAZO B., LEMORINI C., MAKSUD F., MOURRE V., QUINIF Y., THIÉBAUT C. 2005 - Tour-deFaure : grotte de Coudoulous I, Bilan scientifique. Région Midi-Pyrénées, t. 2001, p. 131-133.

JAUBERT J., BRUGAL J.-P., JEANNET M., KERVAZO B. 2005 - Tour de Faure : grotte de Coudoulous I, Bilan scientifique. Région Midi-Pyrénées, t. 2002, p. 129-131.
JAUBERT J., KERVAZO B., BAHAIN J.-J., BRUGAL J.-P. CHALARD P., FALGUĖRES C., JARRY M., JEANNET M., LEMORINI C., LOUCHART A., MAKSUD F., MARTIN H., MOURRE V., QUINIF Y., THIÉBAUT C. 2005 Coudoulous I (Tour-de-Faure, Lot), site du Pléistocène moyen en Quercy: bilan pluridisciplinaire, in: Les premiers peuplements en Europe : colloque international [...], Rennes, 22-25 septembre 2003, Molines N., Moncel M.-H., Monnier J.-L. (Dir.), Oxford, John and Erica Hedges Ltd, p. 227-251 (British archaeological Reports - International Series ; 1364)

JEANNET M. 2005 - La microfaune de l'Igue des Rameaux à Saint-Antonin-Noble-Val (Tarn-et-Garonne, France) : essai de biostratigraphie, Préhistoire du Sud-Ouest, t. 12, 2, p. 109-125.

JEANNET M., BRUGAL J.-Ph. 2006 - The contribution of micro-vertebrates to the description of paleoenvironments, in: 15ème Congrès de l'Union internationale des sciences préhistoriques et protohistoriques, Lisbonne 2006 : Symposium C67 «Settlement dynamics and environement ressources in the Palaeolithic of southwestest France : the case of the Quercy region». Abstracts volume 1, Jarry M., Brugal J.-P., Ferrier C. (Dir.), p. 410-411.

SLIMAK L., BROCHIER J.E., BRUGAL J.-P., COUDENNEAU A., CRAVINHO S., DUTOUR O., GIRAUD Y., GERBE M., JEANNET M., LOMPRÉ A., MALLOL DUQUE C., YVORRA P. 2006 - Installations de la fin du Paléolithique moyen de la grotte Mandrin, Malataverne (Drôme), Lyon / Aix-en-Provence, Rhône-Alpes. Service régional de l'Archéologie / ESEP (UMR 6636), 172 p. (Rapport de fouille programmée annuelle Opération 2006/1111 - Programme P3 - Patriarche 9099) [consulté le 2006.12.14]

VALOUR N., MOKKADEM K., avec la collaboration de BROCHIER J.L., FABRE L., JEANNET M., CECILLON C. 2006 - L'évolution d'un hameau rural du XII ${ }^{e}$ au XV $\mathrm{XV}^{\mathrm{e}} \mathrm{s}$ : Beaume (Chateauneuf sur Isère, Drôme), In: Habitats, nécropoles et paysages dans la moyenne et la basse vallée du Rhône ( $V I^{e}-X V^{e}$ s.) : contribution des travaux du TGV "Méditerranée " à l'étude des sociétés rurales médiévales, Maufras O. (Dir.), Paris, Maison des Sciences de l'Homme, p. 23-37 (Documents d'Archéologie française ; 98).

ARGANT A., ARGANT J., JEANNET M., ERBAJEVA M.A. 2007 - The big cats of the fossil site Château Breccia Northern Section (Saône-et-Loire, Burgundy, France): stratigraphy, palaeoenvironment, ethology and biochronological dating, in: Late Neogene and Quaternary biodiversity and evolution: Regional developments and interregional correlations : proceedings of the 18th international Senckenberg Conference (VI international Palaeontological Colloquium in Weimar) : volume 2, Kahlke R.-D., Maul L.C., Mazza P.P.A. (Dir.), Frankfurt am Main, Schweitzerbart'sche, p. 121-140 (Courier Forschungsinstitut Senckenberg ; 259).

FONTANA L., ARGANT A., LANG L., AUBRY T., CHAUVIĖRE F.-X., DIGAN M., JEANNET M., MANGADO LLACH J., MARCHAL F., MOURER-CHAUVIRÉ C., MÜLLER W., PASQUINI A., SELLAMI F. 2007 - Aide à la 
préparation de publication : Creuzier-le-Vieux : Les Petits Guinards, Bilan scientifique. Région Auvergne 2006, p. 24.

MAUREILLE B., BRUXELLES L., COLONGE D., COSTAMAGNO S., CRAVINHO S., JEANNET M., LAROULANDIE V., THIÉBAUT C., MOURRE V. 2007 Nouveaux vestiges humains moustériens de la grotte du Noisetier (Fréchet-Aure, Hautes-Pyrénées), Bulletins et Mémoires de la Société d'Anthropologie de Paris, t. 19, 3-4 "Résumés des communications et posters de la $1833^{e}$ réunion scientifique de la Société d'Anthropologie de Paris, Hôpital Enfant de La Timone, Marseille, 23-25 janvier 2008 », p. 282-.

ARGANT A., BONNEAU M., JEANNET M., ARGANT T., WIETHOLD J., PROST M., ARGANT J. 2008 - De la diversité des contextes : les os animaux du sanctuaire de Ménestreau (Nièvre) et leur environnement, in: Archéologie du sacrifice animal en Gaule romaine : Rituels et pratiques alimentaires, Lepetz S., Van Andringa W. (Dir.), Montagnac, Monique Mergoil, p. 77-87 (Archéologie des Plantes et des Animaux ; 2).

ARGANT A., JEANNET M., BARRIQUAND J., BARRIQUAND L., ARGANT J. 2008 - Evolution des associations à grands félidés de la Brèche de Château (Bourgogne, France) au cours du Pléistocène moyen : stratigraphie, datation et paléoenvironnement, Stalactite, t. 50,2 , p. 6-9.

ESCUDÉ E., MONTUIRE S., DESCLAUX E., QUÉRÉ J.P., RENVOISÉ E., JEANNET M. 2008 - Reappraisal of " chronospecies " and the use of Arvicola (Rodentia, Mammalia) for biochronology, Journal of archaeological Science, t. 35, 7, p. 1867-1879

http://www.sciencedirect.com/science/article/B6WH84RKDHWM-3/1/968669920240f65b46cf8629d8e0bb43.

FONTANA L., AUBRY T., CHAUVIĖRE F.-X., DIGAN M., JEANNET M., LANG L., MANGADO LLACH J., MARCHAL F., MOURER-CHAUVIRÉ C., MÜLLER W., PASQUINI A., SELLAMI F. 2008 - Aide à la préparation de publication : Creuzier-le-Vieux : Les Petits Guinards, Bilan scientifique. Région Auvergne 2007, p. 26-.

JEANNET M. 2008 - La microfaune de quelques sites tardiglaciaires subalpins in: La fin du Paléolithique supérieur dans les Alpes du nord et le Jura méridional : approches culturelles et environnementales : projet collectif de recherche, Pion G. (Dir.), Paris, Société préhistorique française, p. 87-96, 12 tabl. (Mémoires de la société préhistorique française ; 50).

MOURRE V., COSTAMAGNO S., THIÉBAUT C., ALLARD M., BRUXELLES L., COLONGE D., CRAVINHO S., JEANNET M., JUILLARD F., LAROULANDIE V., MAUREILLE B. 2008 - Le site moustérien de la Grotte du Noisetier à Fréchet-Aure (Hautes-Pyrénées) - premiers résultats des nouvelles fouilles, In: Les sociétés du Paléolithique dans un Grand Sud-Ouest de la France : nouveaux gisements, nouveaux résultats, nouvelles méthodes, Jaubert J., Bordes J.-G., Ortega Cordellat I.
(Dir.), Paris, Société préhistorique française, p. 189-202 (Mémoires de la Société préhistorique française ; 47).

ALLARD M., JUILLARD F., LE GALL O., MARTIN H., JEANNET M. 2009 - Faunes paléolithiques des Peyrugues à Orniac, Lot, Préhistoire du Sud-Ouest, t. 17, p. 141-217.

JEANNET M. 2009 - La micro-faune d'Orniac - Les Peyrugues (Lot) : essai d'écologie quantifiée et chronologie relative, Préhistoire du Sud-Ouest, t. 17, p. 179-197.

JEANNET M. 2009 - L'environnement tardiglaciaire préalpin : essai de restitution basée sur le potentiel climatique et écologique des microvertébrés, Revue archéologique de l'Est, t. 58, p. 5-56 http://rae.revues.org/5499.

JEANNET M., VITAL J. 2009 - La grotte de la ChauveSouris à Donzère (Drôme, France) : L'environnement holocène par la méthode quantitative appliquée aux microvertébrés. Essai de définition et application, Revue de Paléobiologie, t. 28, 1, p. 139-173 http://www.ville-ge.ch/mhng/paleo/paleo-pdf/28-1/pal-28-109.pdf.

ARGANT A., ARGANT J., BARRIQUAND J., BARRIQUAND L., JEANNET M. 2010 - [Poster] The big cats of the Château fossil breccia (Burgundy, France), In: 16th International cave Bear and Lion Symposium : EuroeSpeleoProject $=16$ ème Symposium international de l'Ours et du Lion des Cavernes, Azé (Saône-et-Loire, France), september 22nd-26th, 2010 : Program / Guide Book of excursions = programme $/$ Livret guides des excursions, Argant A. (Dir.), Villeurbanne, Association de Recherche Paléoécologique en Archéologie (ARPA), p. 72.

FONTANA L., AUBRY T., CHAUVIÈRE F.-X., DIGAN M., JEANNET M., LANG L., MANGADO LLACH J., MARCHAL F., MOURER-CHAUVIRÉ C., MUELLER W., PASQUINI A., SELLAMI F. 2010 - Aide à la préparation de publication : Creuzier-le-Vieux : Les Petits Guinards, Bilan scientifique. Région Auvergne 2008, p. 45-46.

GUÉRIN C., avec la collaboration de AFFOLTER J., ARGANT A., ARGANT J., Association ARCH-FAUNE COMTOISE, AUGUSTE P., BAHAIN J.-J., BAILON S., BOULBES N., DETREY J., DURLET C., FALGUĖRES C., FERNANDEZ P., FOSSE P., FOURVEL J.-B., GARCIA T., GAYET J., GOURICHON L., JEANNET M., LABE B., LE GALL O., MALVESY T., MOTTE D., PAUPE P., ROUSSELIĖRES F., SHAO Q., VERCOUTÈRE C. 2010 Le gisement pléistocène moyen récent de Romain-laRoche (Doubs, France) : synthèse biostratigraphique et paléoécologique, In: L'aven pléistocène moyen final de Romain-la-Roche (Doubs, France), Guérin C., Malvesy T. (Dir.), Genève, Muséum d'histoire naturelle, p. 881-895 (Revue de Paléobiologie ; 29/2).

http://www.ville-ge.ch/mhng/paleo/paleo-pdf/292/pal_29_2_07_20.pdf.

JEANNET M. 2010 - Aven de Romain-la-Roche (Doubs, France). Paléoécologie et biostratigraphie selon les microvertébrés, In: L'aven pléistocène moyen final de Romain-la-Roche (Doubs, France), Guérin C., Malvesy T. 
(Dir.), Genève, Muséum d'histoire naturelle, p. 477-493 (Revue de Paléobiologie ; 29/2).

http://www.ville-ge.ch/mhng/paleo/paleo-pdf/292/pal_29_2_07_06.pdf.

JEANNET M. 2010 - L'écologie quantifiée. Essai de description de l'environnement continental à l'aide des microvertébrés = Quantified Ecology. Draft description of the continental environment using microvertebrates, Préhistoires méditerranéennes, t. 1, p. 11-34

http://pm.revues.org/index492.html.

PRIMAULT J., avec la collaboration de BERTHET A.-L., BROU L., DELFOUR G., GABILLEAU J., GRIGGO C., GUÉRIN S., GAMBIER D., HOUMARD C., JEANNET M., LACRAMPE-CUYAUBÈRE F., LANGLAIS M., LAROULANDIE V., LIARD M., LIOLIOS D., LOMPRÉ A., LUCQUIN A., MISTROT V., RAMBAUD D., SCHMITT A., SOLER L., TABORIN Y., VISSAC C. 2010 - La grotte du Taillis-des-Coteaux à Antigny (Vienne) in: Préhistoire entre Vienne et Charente : hommes et sociétés du Paléolithique, Buisson-Catil J., Primault J. (Dir.), Chauvigny, Association des publications chauvinoises, p. 271-293 (Mémoire ; 38).

SLIMAK L., LEWIS J.E., CRÉGUT E., METZ L., OLLIVIER V., ANDRÉ P., CHRZAVZEZ J., GIRAUD Y., JEANNET M., MAGNIN F. 2010 - Le Grand Abri aux Puces, a Mousterian site from the Last Interglacial: paleogeography, paleoenvironment, and new excavation results, Journal of archaeological Science, t. 37, 11, p. 2747-2761

http://www.sciencedirect.com/science/article/B6WH8509XR24-2/2/9f66e4d14dd1967b2362f3ac77934b9e.

SLIMAK L., LEWIS J.E., CRÉGUT E., METZ L., OLLIVIER V., ANDRÉ P., CHZRAZVEZ J., GIRAUD Y., JEANNET M., MAGNIN F. 2010 - The OIS 5e Middle Paleolithic Site of Le Grand Abri aux Puces (Vaucluse, France): Paleogeography, Paleoenvironment, and Initial Results of New Excavations, In: Paleoanthropology Society Meeting Abstracts - St. Louis, Missouri, 13-14 april 2010, Paleoanthropology Society (Dir.), Philadelphia, p. A31- (PaleoAnthropology 2010).

http://www.paleoanthro.org/journal/content/PAS2010A.pdf.

ARGANT A., ARGANT J., BARRIQUAND J., BARRIQUAND L., BONNEFOY M., GUILLOT L., JEANNET M. 2011 - Pleistocene Carnivores in the Mâconnais. Field-trip of the 16th International Cave bear and Lion Symposium (Azé, 2010), in: Proceedings of the 16th International Cave Bear and Lion Symposium, Azé (Saône-et-Loire, France), 22-26 septembre 2010, Argant A., Argant J. (Dir.), Paris, Association Française pour l'Etude du Quaternaire, p. 27-37 (Quaternaire ; H.S 4).

BARRIQUAND L., BARRIQUAND J., ARGANT A., FLOSS H., GALLAY A., GUÉRIN C., GUILLOT L., JEANNET M., NYKIEL C., QUINIF Y. 2011 - Le site des Grottes d'Azé, in: Proceedings of the 16th International Cave Bear and Lion Symposium, Azé (Saône-et-Loire, France), 22-26 septembre 2010, Argant A., Argant J. (Dir.), Paris, Association Française pour l'Etude du Quaternaire, p. 1525 (Quaternaire ; H.S 4).
GAMBERI ALMENDRA DE CARVALHO L., ARGANT A., ARGANT J., BARTH P., BOUDADI-MALIGNE M., BOULBES N., BRUGAL J.-P., CARAMELLI D., CONDEMI S., CRÉGUT E., DEBARD E., ERRERA M.G.L., FARRE B., FAURE M., FERNANDEZ P., GEIGL E.-M., GUÉRIN C., HARTER S., JEANNET M., LATEUR N., MALLYE J.-B., MARTIN S., MONNEY J., ROMAN C., ROUSSELIĖRES F., SABAUT M., VALLI A. 2011 - L'aven de l'Arquet Barjac (30) : étude d'un aven piège, Ardèche Archéologie, t. 28 , p. 3-10.

OPPLIGER J., JEANNET M., MORALA A., STUDER J., BESSE M. 2011 - Les micromammifères (Chiroptera, Soricomorpha et Rodentia) du gisement tardiglaciaire et holocène du Moulin du Roc (St-Chamassy, Dordogne, France) : implications paléo-environnementales, Revue de Paléobiologie, t. 30, 1, p. 123-132.

JAUBERT J., BRUGAL J.-P., VENDITTI F., HERNANDEZ M., BOUDADI-MALIGNE M., ARGANT A., BAHAIN J.-J., COUCHOUD I., FALGUERES C., FERNANDEZ P., JEANNET M., KERVAZO B., LATEUR N., LE GALL O., LÉCUYER C., LEMORINI C., MARTIN H., MERCIER N., MOURRE V. 2012 - Coudoulous I in Quercy (SW France): from marginal scavenging to a kill-butchery site for Preneandertal, in: 2nd Annual Meeting, Bordeaux, September 21-22, 2012 : Abstracts, European Society for the study of Human Evolution (Dir.), Bordeaux, ESHE, p. 88.

MOURRE V., GERBE M., DELFOUR G., BRUXELLES L., COLONGE D., COUDENNEAU A., CRAVINHO S., GUILLERMIN P., JEANNET M., LAROULANDIE V., MAUREILLE B., MICHEL A., THIÉBAUT C. 2012 [Abstract] Les Fieux (Lot, France) : paleolithic levels, report of new studies, in: 54th annual Meeting Toulouse : 10th14th of april, 2012, Hugo-Obermaier Society for Quaternary Research and Archaeology of Stone Age (Dir.), Erlanger, Hugo-Obermaier-Gesellschaft für Erforschung des Eiszeitalters und der Steinzeit, p. 39-

ARGANT T., DJERBI H., GUÉRIN B., JEANNET M., LATOUR-ARGANT C. 2013 - Le puits de la Logère à Anse (Rhône) - Un exemple de biodiversité antique et ses implications paléoenvironnementale et paléoclimatique. Bulletin mensuel Société Linnéenne de Lyon, 2013, 82 (910), p. 255-269.

JEANNET M. 2013 - Les soricidés (Insectivora, Mammalia) de la Grotte du Noisetier à Fréchet (Hautes-Pyrénées, France) : recherche de la Musaraigne ibérique (Sorex granarius Miller, 1910). Revue de paléobiologie, Genève, 32 (1) : 243-247.

JEANNET M., MOURRE V. 2013 - Mimomys pyrenaicus n.sp., nouvel arvicolidé (Mammalia, Rodentia) dans le Pléistocène supérieur des Pyrénées (Fréchet-Aure, Hautes-Pyrénées, France), Paleo, t. 24, p. 139-147 http://paleo.revues.org/2570

JEANNET M., BRUGAL J.-P., JARRY M. 2013 - Microfaune et paléoclimatologie dans le Pléistocène moyen et supérieur du Quercy. Essai d'écologie quantifiée, in: Modalité d'occupation et exploitation des milieux au Paléolithique dans le Sud-Ouest de 
la France : l'exemple du Quercy, Jarry M., Brugal J.-P., Ferrier C. (Dir.), Les Eyzies-de-Tayac, Société du Musée national de préhistoire et de la recherche archéologique, p. 107-144 (Paléo. Supplément ; 4 / Actes du $15^{\circ}$ Congrès de l'Union internationale des sciences préhistoriques et protohistoriques, Lisbonne 2006 - Session C67).

ROYER A., LÉCUYER C., MONTUIRE S., AMIOT R., LEGENDRE S., CUENCA BESCÓS G., JEANNET M., MARTINEAU F. 2013 - What does the oxygen isotope composition of rodent teeth record?, Earth and Planetary Science Letters, t. 361, Supplement C, p. 258-271 http://www.sciencedirect.com/science/article/pii/S0012821 X12006061.

CASTEL J.-C., BOUDADI-MALIGNE M., WITH THE COLLABORATION OF, BIGNON O., BRUGAL J.-P., CAMUS H., CHAUVIÈRE F.-X., DUCASSE S., GUADELLI J.-L., KUNTZ D., JEANNET M., LAROULANDIE V., MALLYE J.-B., RENARD C. 2014 - The contribution of dated paleontological sites to the understanding of human behaviours and their environment. The pit fall of the Igue du Gral and the Upper Paleolithic of eastern Aquitaine, in: 12th ICAZ Congress, 22-27th Sept, Mendoza, Argentine.

DAVID S., ROBERT É., PIGEAUD R., D'ERRICO F., BEREIZIAT G., CAILHOL D., GRIGGO C., JAILLET S., JEANNET M., NATON H.-G., PAITIER H., PETROGNANI S. 2014 - La grotte des Gorges (Jura) : un site inédit à l'interface des territoires symboliques du Paléolithique supérieur ancien, In: Modes de contacts et de déplacements au Paléolithique eurasiatique = Modes of contact and mobility during the Eurasian Palaeolithic, Otte M., Le Brun-Ricalens F. (Dir.), Liège / Luxembourg, Université de Liège / Musée National d'Histoire et d'Art, p. 537-567 (Etudes et Recherches archéologiques de l'Université de Liège (ERAUL) ; 140 / ArchéoLogiques ; 5).

GERBE M., THIÉBAUT C., MOURRE V., BRUXELLES L., COUDENNEAU A., JEANNET M., LAROULANDIE V. 2014 - Influence des facteurs environnementaux, économiques et culturels sur les modalités d'exploitation des ressources organiques et minérales par les Néandertaliens des Fieux (Miers, Lot), In: Transitions, ruptures et continuité en Préhistoire : [Volume 2 : Paléolithique et Mésolithique], Jaubert J., Fourment N., Depaepe P. (Dir.), Paris, Société préhistorique française, $p$. 257-279 (Congrès préhistorique de France. Compte Rendu de la 27ème Session, Bordeaux 2010 [Session E]).

ROYER A., LÉCUYER C., MONTUIRE S., PRIMAULT J., FOUREL F., JEANNET M. 2014 - Summer air temperature, reconstructions from the last glacial stage based on rodents from the site Taillis-des-Coteaux (Vienne), Western France, Quaternary Research, t. 82, 2, p. 420-429

http://www.sciencedirect.com/science/article/pii/S0033589 414000830.
ROYER A., MONTUIRE S., LEGENDRE S., DISCAMPS E., JEANNET M., LÉCUYER C. 2016 - Investigating the Influence of Climate Changes on Rodent Communities at a Regional-Scale (MIS 1-3, Southwestern France), PLoS ONE, t. 11, 1, p. e0145600 http://dx.doi.org/10.1371\%2Fjournal.pone.0145600.

JEANNET M., MEIN P. 2016 - Les Muridae (Mammalia, Rodentia) du Pléistocène moyen de l'lgue des Rameaux (Tarn-et-Garonne, France), Paleo, t. 27, p. 177-205.

JEANNET M. (soumis en 2017) - Vergisson II (Saône-etLoire, France) : microfaune et environnement de l'Homme de Néanderthal. Quaternaire, $n^{\circ}$ spécial Hommage à Cl. Guérin.

GRIGGO C., BERNARD-GUELLE S., TILLET T., ARGANT A., JEANNET M. (soumis en 2017) - La grotte de Prélétang (Presles, Isère) : Un camp de chasse moustérien de moyenne montagne. Actes de la Table Ronde AVDPA, L'Homme dans les Alpes, de la Pierre au Métal, Villard-deLans, 13-15 Octobre 2016. EDYTEM.

JEANNET M. (à paraître) - La microfaune de la Grotte Mandrin. Son implication dans l'environnement et la biostratigraphie. Monographie « La Grotte Mandrin (Malataverne, Drôme) 》, L. Slimack (ss. la dir), Préhistoire Méditerranéenne.

JEANNET M. (à paraître) - La microfaune de Coudoulous I paléoenvironnement et biostratigraphie selon les microvertebrés. In : J. Jaubert et al. (dir), Monographie sur Coudoulous I (Lot).

JEANNET M. (à paraître) - Le Potentiel éco-climatique de Pliomys lenki Heller, 1930 (Arvicolidae : Rodentia, Mammalia). Essai de reconstitution.

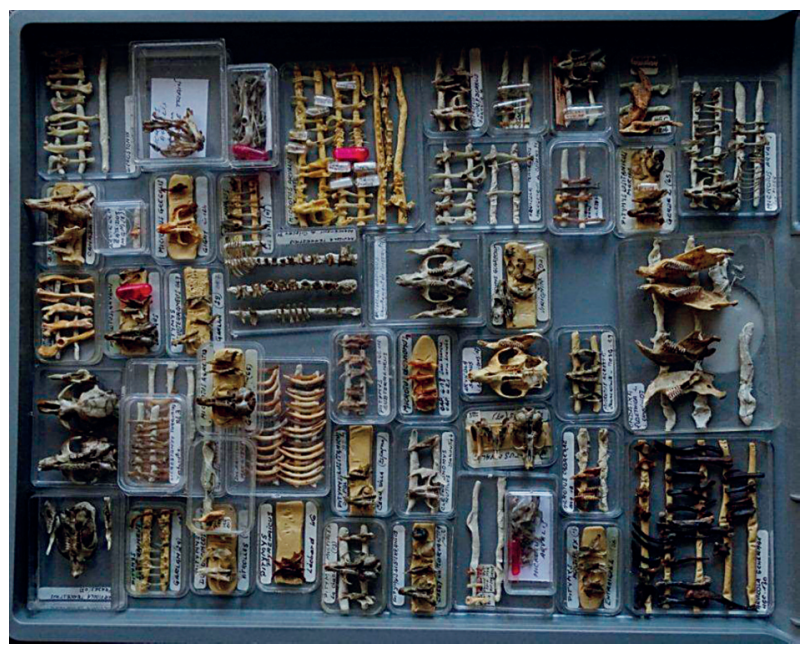

Exemple de tiroir de la collection de comparaison de Marcel Jeannet. 